\title{
El empoderamiento de la mujer como fuente de dignificación social
}

The empowerment of women as a source of social dignification

\author{
Martha Alicia Romero Echevarría* \\ Escuela Profesional de Ciencias de la Comunicación, \\ Universidad de San Martín de Porres, Perú
}

\section{Resumen}

El término empoderamiento fue acuñado en la Conferencia Mundial de Mujeres en Beijing para promover acciones que solventen la dignidad de las mujeres; teniendo esto como base para construir la paz, prosperidad y sostenibilidad (ONU Mujeres, 2015). En el presente estudio, se revisan los alcances o accesibilidad de las mujeres a la educación, economía, sociedad y política, debido a que se considera que una persona empoderada debe alcanzar equilibrio, autonomía y capacidad de actuar según su propio proyecto de vida. El empoderamiento minimiza los actos violentos contra los miembros de la familia y abre caminos para fortalecer y lograr una sociedad más digna.

Palabras clave: empoderamiento, educación, economía, cuidado, familia.

\section{Abstract}

The term "empowerment" was coined at the World Conference on Women held in Beijing, aimed at promoting actions that solve women's dignity, as a basis for building peace, prosperity and sustainability (UN Women, 2015). This study reviews the approachability or accessibility of women to education, economy, society and politics, since it is considered that empowered people

Este es un artículo Open Access bajo la licencia Creative Commons Atribución-NoComercial-Compartirlgual 4.0 
should achieve balance, autonomy and the ability to act according to their own life project. Empowerment minimizes violent acts against family members and opens ways to strengthen and achieve a more dignified society.

Keywords: empowerment, education, economy, care, family.

\section{Introducción}

Desde 1948, en la Declaración Universal de los Derechos Humanos, se estableció que todas las personas tienen derechos inalienables, tanto hombres como mujeres, sin distinción individual o social de alguna índole. En esta declaración «los Estados Miembros han renovado y reafirmado constantemente sus compromisos con la igualdad de género y el empoderamiento de las mujeres en los acuerdos internacionales» (Oficina de las Naciones Unidas Contra la Droga y el Delito [UNODC], 2019, p. 8).

En la Conferencia Mundial de Mujeres en Beijing se acuña el término empoderamiento y se inician acciones para que las mujeres recobren su dignidad; esto debido a las múltiples protestas generadas por el trato diferenciado en la vida social, económica y política; además, se puso énfasis en la necesidad de acciones para la eliminación de la violencia contra la mujer (ONU Mujeres, 2015). El empoderamiento de la mujer abre los caminos para tomar decisiones y vincularse con el poder económico, social y político; ser partícipe de la vida pública.

La Organización Mundial de la Salud (OMS, 2013) declaró como problema de salud pública la violencia contra la mujer, debido a los daños biopsicosociales que se ocasionan por el incremento de los actos violentos en el mundo. Se registra que «el 35\% de las mujeres experimentan violencia y que la proveniente del esposo o la pareja íntima es la más común, abarcando el 30\% de los casos» (párr. 1). Razón por la que se conminó a los Estados a tomar medidas para su erradicación, desde entonces, muchos países asumieron el compromiso de controlar y erradicar estos actos, con sanciones cada vez más duras en protección a las víctimas de la violencia de género. 
En el Perú, el panorama no es diferente. El Estado, en octubre de 1996 creó el Ministerio de Promoción de la Mujer y del Desarrollo Humano (PROMUDEH); luego en julio de 2002, el Ministerio de la Mujer y Desarrollo Social (MINDES); y es a partir de 2012 que pasa a llamarse Ministerio de la Mujer y Poblaciones Vulnerables (MIMP, 2020). Tiene como objetivos primordiales promover la igualdad de oportunidades, proteger a la mujer contra la violencia, trabajar por su desarrollo integral y mejorar las oportunidades, así como generar políticas públicas para la erradicación y control de la violencia de género, con énfasis en la mujer.

El MMIP viene tomando acciones para disminuir las brechas y lograr la igualdad; son múltiples los programas en vigencia, para erradicar la violencia y prevenirla, pero «es necesario contar con un enfoque integrado de gobernabilidad que permita administrar los proyectos de intervención con programas eficientes, oportunos y sostenibles» (Romero, 2019, p. 75). No obstante, la falta de articulación de los organismos y entidades responsables al poner en marcha estos programas afecta el empoderamiento de las personas y de la sociedad.

En este trabajo, se desarrollan los alcances del empoderamiento educativo, económico y social; los aspectos necesarios para un empoderamiento sostenible e inclusivo, sobre la base de la ética y la psicología, valorando las normas y leyes. No como imposición arbitraria sino como responsabilidad compartida que permita una coexistencia digna; es decir, que se actúe por convicción, asumiendo compromisos para el bienestar personal, familiar, social y político.

\section{Estragos de la violencia en la salud física y mental}

La violencia contra la mujer es el resultado de la desigualdad que por mucho tiempo fue admitida en las diferentes sociedades; donde se aceptaron malos tratos, que aún persisten justificados por la costumbre, en especial, donde prima el machismo. Violencia atribuída a la necesidad de usar la fuerza con un carácter opresivo para marcar las desigualdades y la supremacía en la persona. 
La violencia de género se expresa de acuerdo a las experiencias de vida adquiridas en el proceso de socialización; pasan a ser parte de la historia de la persona. Según Ramos-Lira (2014):

[son] conductas violentas, realizadas impulsivamente y motivadas por sentimientos de ira y rabia, que por lo regular se dirige hacia quienes tienen menos poder, es decir, hacia quienes se juzga como inferiores por razones de género o edad. (p. 278)

La violencia física contra la mujer va desde empujones o empellones y manotazos, puñetes, patadas, agresiones con objetos contundentes y armas, que pueden generar lesiones leves o graves, causantes de invalidez parcial o total y llega, en muchos casos, a su expresión máxima que es el feminicidio. En 2019 el Centro de Emergencia Mujer (CEM, 2019, p. 1) registró 166 casos de feminicidio, 404 casos de tentativa de feminicidio y 181885 casos de violencia contra la mujer.

No se cuenta con datos exactos, debido a que la mayoría de las mujeres sometidas a maltrato físico no acuden a los centros de salud por diversos motivos: no lo cree necesario (44.8\%); le da vergüenza (15.9\%); desconocimiento del lugar donde debe ir (13\%); tienen miedo de ser nuevamente golpeada $(8.4 \%)$; causarle algún problema a quien la golpeó (5.4\%); se siente culpable (3.7\%); de nada sirve (3.0\%); el miedo al divorcio o separación $(2.7 \%)$; otras razones $(1.7 \%)$; son cosas de la vida $(1.2 \%)$, según cifras del Instituto Nacional de Estadística e Informática (INEI, 2020, p. 302).

El sometimiento a la violencia es progresivo, pasa de una forma a otra con bastante facilidad, es decir, de la violencia psicológica a la violencia física y a la sexual; desde la denigración hasta la violación y el feminicidio.

La persona que es víctima de violencia sufre estragos emocionales de primer orden, pierde el sentido de vida y otorga su poder y valía personal a la persona que la somete, quedando desprotegida de sus propios recursos para hacer frente a las situaciones; huye del dolor emocional y bloquea toda posibilidad de buscar soluciones al problema de la violencia, lo que denigra su dignidad; en suma, pierde de vista su proyecto de vida. Como consecuencia, se desencadenan problemas de salud mental; se evidencian 
daños psíquicos como ansiedad, depresión, disfunción sexual, trastornos alimenticios, de personalidad, obsesivo compulsivos; intentos de suicidio $\mathrm{u}$ homicidio, en muchas ocasiones con desenlaces mortales (García-Moreno, 2000, p. 12).

\section{El empoderamiento educativo}

La educación es la base del desarrollo personal, social, económico y político ya que conduce a las sociedades a empoderarse de manera evidente. La realidad en el Perú es otra, a pesar de tener políticas públicas destinadas a alcanzar su máximo desarrollo, no se evidencian resultados positivos; existen brechas en este sector que van desde una educación diferenciada económicamente. Los centros educativos públicos no reúnen las condiciones para albergar a la mayoría de los estudiantes del país, y no ofrecen los mismos servicios. Se ha caído en el negocio educativo: centros particulares con diversas ofertas educativas en los tres niveles de educación: básica, secundaria y superior.

En la Ley General de Educación, N. ${ }^{\circ} 28044$, se indica que la educación es un derecho fundamental garantizado por el Estado y que es la sociedad la responsable de velar por el cumplimiento. Sin embargo, existen marcadas desigualdades socioeconómicas, es un país multicultural, plurilingüe, y a esto se suma la primacía del machismo que acentúan las brechas educativas, sobre todo en las zonas rurales y urbano marginales, debido a que se prioriza el trabajo para la subsistencia desde edades tempranas, quedando la educación postergada.

Por lo tanto, es necesario seguir trabajando de manera conjunta con estrategias para empoderar la educación, con igualdad de oportunidades desde las aulas; estimulando la capacidad de discernimiento y la toma de decisiones. En este sentido, Di Laura (2015, coordinadora del Centro de la Mujer de Centrum Católica), señala que si las mujeres son más preparadas académicamente se mejora su perspectiva de vida en el campo familiar, laboral, económico y político.

En la actualidad, han disminuido las distinciones de género para el acceso a la educación, pero las brechas económicas aún subsisten y son estas las 
que se interponen. A pesar de ello, se encuentran ofertas educativas de todo nivel para desarrollar las competencias en el campo elegido y sin distinciones de género para estudiar carreras técnicas o profesionales. Las leyes y políticas públicas en torno a la educación no hacen ningún tipo de distinción en cuanto a género -brechas que no son visibles-.

Definitivamente, es importante el empoderamiento educativo, debido a que «la educación, tanto del hombre como de la mujer, es un factor importante para el empoderamiento femenino» (Glave, 2016, p. 51). Mientras más nivel de educación tengan las personas se verá un mejor trato de sí mismo, cumplimiento de normas, cuidado de los miembros de la familia, del trabajo y de la sociedad; con más opciones para tomar decisiones responsables y seguras.

\section{El empoderamiento económico}

En cuanto a la economía, la mujer viene asumiendo un rol diferenciador, en la actualidad un gran porcentaje de mujeres son económicamente activas, contribuyendo a la economía de las familias y por ende del país. En un estudio realizado en Colombia se hace la diferenciación de género en cuanto a cómo gastan. Se develó que las mujeres gastan un alto porcentaje de su sueldo en salud y educación, mientras que los hombres invierten menos de la mitad en esos rubros (Botello-Peñaloza y Guerrero-Rincón, 2017).

El Fondo Monetario Internacional (FMI, 2019, p. 5) señala que el empoderamiento económico de la mujer es necesario, primero por respeto a la ética, y también porque es una oportunidad para la «búsqueda de la estabilidad macroeconómica y el crecimiento inclusivo», ya que se incrementa la productividad y se tiende al crecimiento económico de hasta un 35\% del PBI, lo que redunda en el crecimiento social.

En el Perú, hace 50 años el 30\% de mujeres trabajaba, 20 años después el $50 \%$ y el 2015 se incrementó a 78\%, repercutiendo en el país a nivel económico, político y social (Di Laura, 2015). En muchos casos las mujeres asumieron emprendimientos por iniciativa propia, creando oportunidades, aprovechando sus capacidades o habilidades; en otros casos por la necesidad de asumir su propia economía y la de su familia. En el Estado se cuenta con programas 
asistenciales y de empoderamiento dirigidos por el MIMP, que a su vez cuentan con el apoyo de la Cooperación Internacional, Organizaciones no gubernamentales y diferentes entidades privadas. Estos diversos proyectos vigentes promueven que las mujeres desarrollen habilidades y destrezas en los campos ocupacionales; en trabajos dependientes para empresas o entidades públicas ocupando puestos o cargos asignados y por el cual reciben una remuneración mensual; y trabajos independientes, en los que se organizan grupos de personas que asumen retos emprendedores.

En la guía práctica para la acción del sector privado, se pone énfasis en el hecho que aún no se ha logrado el posicionamiento de la mujer en el sector empresarial. Se pide a las empresas que trabajen en la misma línea, para lograr los objetivos de desarrollo sostenible, «en las oportunidades para conseguir un trabajo digno y en la representación en los procesos de adopción de decisiones políticas y económicas» (Pacto Mundial de Naciones Unidas, 2016, p. 22). Por tanto, es necesario insistir en el empoderamiento de las mujeres apoyando los emprendimientos, ya que se ha visto que la independencia económica, mediante el trabajo digno es una fuente de fortalecimiento, con ello lo que se busca es que se equiparen los roles de género para lograr el equilibrio social.

\section{El empoderamiento social}

El ser humano es un ser social, desde su concepción hasta su muerte, por lo tanto, debe prepararse para convivir de manera saludable con las demás personas.

El primer despertar social es dentro de la familia, que acoge y forma a los ciudadanos que conforman el país, pero esta concepción fue cambiando con el tiempo y debido a los diferentes momentos vividos en la historia. Las formas y tipos de familia se han diversificado. Uno de los grandes motivos es la violencia ejercida al interior de las familias, con consecuencias sociales funestas. A decir de Leuridan (2019): «la destrucción de la familia traería la destrucción de la sociedad porque al ser humano le faltaría la educación de pertenencia a otros que lo quieren» (p. 279). Esta falta de pertenencia y de amor por la familia se evidencia cuando las noticias muestran a personas, 
que en tiempos de pandemia, salen a las calles a divertirse desenfrenadamente incumpliendo con las normas de bioseguridad sanitaria, y más aún, regresan a sus casas con el riesgo de contagiar a su familia sin que esto les importe.

Por otra parte, muchos jóvenes no piensan en la familia y asumen el sexo libre sin protección, pensando en el placer momentáneo, procreando un ser que queda expuesto a la poca planificación y preparación para el cuidado y crianza de un niño. En otros casos, personas que se acercan al matrimonio con una consigna egoísta que se ha vuelto común, no existe conciencia de lo que significa, es condicional: «si en el matrimonio me va bien me quedo, pero si me va mal me divorcio, total hay solución para todo».

La escuela es otro espacio de desarrollo social. Si bien, es cierto que las políticas educativas apoyan que todos los niños y niñas tienen la misma oportunidad de acceder a la educación, lo que se evidencia es que en el país aún hay mucho que trabajar para lograr ciudadanos valiosos, esto debido a que en una sociedad donde predomina la desconfianza y la inseguridad social, las manifestaciones de los modelos de violencia se replican en las escuelas. Por eso, es necesario enseñar desde una perspectiva integral.

Cuando una persona que ha vivido en violencia de manera sistemática y por diferentes motivos logra romper con estos esquemas, puede evaluar las situaciones vividas y las «puede afrontar mediante el proceso potenciador de concientización» (Martín, 2012, p. 11). A partir de este momento de ruptura, puede encaminarse hacia la búsqueda del equilibrio personal, abriendo las puertas al conocimiento y al emprendimiento.

Las mujeres se han empoderado socialmente progresivamente, con roles cada vez más visibles y decisivos en los diferentes espacios de desarrollo, ya sea rural, urbano, local o nacional. Sin embargo, todos estos aspectos que deberían empoderar a la sociedad para lograr el equilibrio no se pueden visibilizar. Las diferentes manifestaciones de la violencia siguen apareciendo, sin tener en cuenta edad, educación o condición socioeconómica.

Por consiguiente, surge la necesidad de intervenir en el fortalecimiento de las habilidades blandas de las personas, especialmente de la mujer; de manera que se empodere, sienta más seguridad, equilibre su autoestima, 
potencie su inteligencia emocional y desarrolle la comunicación asertiva y su capacidad de resiliencia.

Si se logra la igualdad de género, se logrará paz, prosperidad y un mundo sostenible. Es necesario «asegurar la participación plena y efectiva de las mujeres y la igualdad de oportunidades de liderazgo a todos los niveles decisorios en la vida política, económica y pública» (Cepal, 2016, p. 17). Trabajar en función de los determinantes sociales; asumiendo compromisos personales y sociales se reducirá la violencia.

\section{El empoderamiento axiológico}

El empoderamiento tiene como objetivos el desarrollo personal, aportar en la calidad de vida, y de esta forma, fortalecer la dignidad de todas las personas.

El psicólogo norteamericano Lawrence Kohlberg, entre los años 1981 y 1984, investigó sobre desarrollo moral. Presentó una serie de dilemas morales a niños y hombres; identificó seis etapas de desarrollo moral que se equiparan con las etapas de desarrollo de Piaget. En ese estudio no participaron mujeres, considerando que no responderían de manera objetiva; se provoca una dicotomía adscrita a los espacios de la construcción de los géneros, donde las mujeres no son capaces de elaborar juicios de orden superior por haber sido emplazada por la sociedad a la esfera privada y doméstica (como se citó en Medina-Vicent, 2016).

En contraste, la psicóloga Carol Gilligan, discípula de Kohlberg, establece que las mujeres no solo son capaces de elaborar juicios de orden superior, sino que además, poseen la capacidad innata de estar en permanente cuidado de las otras personas, priorizando las necesidades para darle a cada cual lo que necesita. La ética del cuidado busca que todos los seres humanos, sin distinción de ningún tipo, asuman responsabilidad y solidaridad en todos los ámbitos, como la familia, la amistad, la política y las relaciones sociales (como se citó en Borgeaud-Garciandía, 2020).

Gilligan coincide con Kohlberg en la necesidad de tratar a todas las personas con igualdad, pero la ética de justicia busca la imparcialidad y el trato igualitario, mientras que la ética del cuidado respeta la diversidad para 
que las personas asuman la convivencia con respeto a la diversidad, destacando «el rol de la responsabilidad, no desde la óptica liberal de la justicia, sino como un elemento que desarrollan los actores morales insertos en relaciones concretas de interdependencia» (como se citó en BorgeaudGarciandía, 2020, p. 43).

Leuridan nos ilustra en La ética de las virtudes. Destaca la responsabilidad del ser humano para actuar con conciencia teniendo en cuenta que existen leyes y normas comunes a todos, lo que no significa que todos las cumplan por convicción; estableciendo la diferencia en las personas que hacen el bien por convicción. «Se trata de formar una actitud desde la cual se analizará y se tomará decisiones. La acción correcta depende de la persona que tiene la actitud correcta» (Leuridan, 2015, p. 129).

Es necesario que cada persona tenga un proyecto de vida, que le permita aplicar sus capacidades y desarrollar sus potencialidades para el progreso personal y social, asumiendo el compromiso de elaborar estrategias "para promover la igualdad de género y el empoderamiento de las mujeres (UNODC, 2019, p. 8).

En consecuencia con lo dicho, el empoderamiento implica tomar conciencia de la necesidad de contar con personas justas, que asuman la responsabilidad de una coexistencia digna y que actúen por convicción; para ello, es necesario que todos los ciudadanos asuman un compromiso por el bienestar personal, familiar, y por ende, de la sociedad.

\section{A modo de conclusión se plantean estrategias para el empoderamiento}

Es necesario empoderar a las mujeres con estrategias de afrontamiento. Se cuenta con los centros de salud del Estado, las organizaciones sin fines de lucro y los grupos de ayuda psicológica que, de manera voluntaria, atienden a nivel nacional. La finalidad es mejorar la calidad de vida, sobre todo en este contexto de crisis sanitaria que se vive por la pandemia mundial provocada por la COVID-19.

Este empoderamiento se puede lograr mediante talleres, donde las propias mujeres tomen conciencia y amplíen su campo perceptual, para 
que puedan visibilizar un mejor futuro, con calidad de vida personal y familiar. De allí lo trascendente de trabajar en sus capacidades y en la gestión de un proyecto de vida personal. Asimismo, orientarlas al autodiagnóstico que les permita encontrar sus fortalezas internas, sus debilidades y sus propios temores; así como establecer estrategias claras y fortalecer sus recursos, con una actitud proactiva, minimizando los miedos para enfrentar la vida.

En este sentido, lograr la autonomía: el cuidado del yo, es el primer pilar planteado por Gilligan (como se citó en Borgeaud-Garciandía, 2020). Se asegura la supervivencia, es la base de la realización personal, se trabaja el autoconocimiento, el autorrespeto y la autoconciencia; le ayuda a tener seguridad y fortalecer su propia estima y valía personal. El aprecio por sí misma cambia su autopercepción, toma decisiones libremente.

El segundo pilar es el cuidado del otro. Se asume la responsabilidad, inherente a la naturaleza de la mujer, siempre está cuidando y protegiendo a otros seres humanos, dándole a cada cual lo que necesita. Solamente es posible si se tiene como prioridad el cuidar de sí misma. Se afianza el respeto por los demás y el compromiso de trabajar juntos por el bienestar (Gilligan, como se citó en Borgeaud-Garciandía, 2020).

La inclusión del yo y de los otros, es el tercer pilar. Corresponde a la responsabilidad en el cuidado de la sociedad, comunidad, entorno, incluso el medioambiente, para asegurar una vida más saludable; aplicando principios que son aceptados socialmente. Estando en compromiso con los demás, trabajando juntos para lograr una sociedad mejor, respetando las diferencias y necesidades individuales, pensando y actuando por el bien común (Gilligan, como se citó en Borgeaud-Garciandía, 2020).

Finalmente, el enfoque en el empoderamiento, que se realiza en el presente trabajo, obedece a la necesidad de fortalecer las estructuras psíquicas para lograr una igualdad de género. Tanto hombres y mujeres merecen un trato digno; por lo que juntos deben asumir la responsabilidad de construir una sociedad mejor. 


\section{Referencias}

Borgeaud-Garciandía, N. (2020). Cuidado y responsabilidad. Estudos Avançados, 34(98), 41-56. https://doi.org/ 10.1590/s0103-4014.2020.3498.004

Botello-Peñaloza, H. A. y Guerrero-Rincón, I. (2017). Condiciones para el empoderamiento de la mujer rural en Colombia. Entramado, 13(1), 62-70.

Cepal. (2016). Agenda 2030 y los objetivos de desarrollo sostenible. Una oportunidad para América Latina y el Caribe. Publicación de las Naciones Unidas.

Di Laura, G. (2015, 25 de junio). El aporte de la mujer al desarrollo del país. Gestión [Economía]. https:/l gestion.pe/economia/aporte-mujer-desarrollo-pais-93305-noticia/

Fondo Monetario Internacional (FMI). (2019). Las mujeres y el crecimiento económico. Finanzas \& Desarrollo (56), 1. https://www.imf.org/external/pubs/ft/fandd/spa/2019/03/pdf/fd0319s.pdf

García-Moreno, C. (2000). Violencia contra la mujer: género y equidad en la salud. Harvard Center for Population and Development Studies. https://iris.paho.org/hitstream/handle/10665.2/811/ 9789275327166. pdf?sequence $=1$

Glave, C. R. (2016). Cambios en el empoderamiento de la mujer: el caso de las beneficiarias del Programa Juntos en el Perú, 2014 [Tesis de licenciatura, Pontificia Universidad Católica del Perú].

Instituto Nacional de Estadística e Informática (INEI). (2020). Encuesta demográfica y de salud familiar, 2019. https://www.inei.gob.pe/media/MenuRecursivo/publicaciones_digitales/Est/Endes2019/Libro.pdf

Leuridan, J. (2015). La ética de las virtudes. Cultura, (29), 127-174.

Leuridan, J. (2019). El sentido de las dimensiones éticas de la vida. USMP, Fondo editorial.

Martín, A. (2012). Proyecto de intervención para el empoderamiento de las mujeres de guardo. Escuela universitaria de Palencia "La Yutera». https://uvadoc.uva.es/bitstream/handle/10324/2080/TFGL\%20118.pdf?sequence=1\&isAllowed=y

Medina-Vicent, M. (2016). La ética del cuidado y Carol Gilligan: Una crítica a la teoría del desarrollo moral de Kohlberg para la definición de un nivel moral postconvencional contextualista. Daímon, Revista Internacional de Filosofía, (67), 83-98. https://doi.org/10.6018/199701

Ministerio de la Mujer y Poblaciones Vulnerables (MIMP). (2020). Reporte estadístico de casos con características de feminicidio registrados por los centros emergencia mujer. https://www.mimp.gob.pel contigo/contenidos/pncontigo-articulos.php?codigo=39

Oficina de Naciones Unidas contra la Droga y el Delito (UNODC). (2019). Estrategia para la igualdad de género y el empoderamiento de las mujeres (2018-2021). Sección de Servicios en inglés, Publicaciones y Biblioteca, Oficina de las Naciones Unidas en Viena.

Organización Mundial de la Salud (OMS). (2013, 20 de junio). Informe de la OMS destaca que la violencia contra la mujer es "un problema de salud global de proporciones epidémicas". Centro de prensa. https:/l www.who.int/mediacentre/news/releases/2013/violence_against_women_20130620/es/ \#: :text=20\%20de\%20junio\%20de\%202013

ONU Mujeres. (2015). La Plataforma de Acción de Beijing: inspiración entonces y ahora. https:/l beijing20.unwomen.org/es/about

Pacto Mundial de Naciones Unidas. (2016). El sector privado ante los ODS. Guía práctica para la acción. Educamet.

Ramos-Lira, L. (2014). ¿Por qué hablar de género y salud mental? Salud Mental, 37(4), 275-281. 